\title{
Padrões da Distribuição Nictemeral do Comportamento Ingestivo de Vacas Leiteiras, ao Início e ao Final da Lactação, Alimentadas com Dieta à Base de Silagem de Milho
}

\author{
Vivian Fischer ${ }^{1}$, Armand Gerard Deswysen ${ }^{2}$, Pierre Dutilleul ${ }^{3}$, Johan De Boever ${ }^{4}$
}

\begin{abstract}
RESUMO - Os efeitos dos estádios de lactação inicial e final sobre o comportamento e a distribuição nictemeral do comportamento ingestivo foram avaliados com oito vacas leiteiras, consumindo uma dieta à base de silagem de milho. A análise espectral das séries de tempos horários de mastigação (ingestão ou ruminação) foi realizada usando os dados modificados pela transformação finita de Fourier. Foi realizada análise de variância destes dados e a análise dos periodogramas e diagramas de fase. A duração média das refeições e o consumo de matéria seca foram superiores, enquanto o tempo unitário de ruminação foi inferior no período inicial em relação ao final da lactação. A distribuição dos tempos de ingestão e de ruminação nos estádios inicial e final de lactação seguiu, respectivamente, periodicidades de 24, 12, 8 e 4 horas e de 24, 12 e 8 horas. Foi constatado efeito significativo do estádio de lactação para o tempo gasto ingerindo e ruminando no componente de ritmo 4 . O estádio da lactação influenciou alguns parâmetros do comportamento ingestivo e sua ritmicidade.
\end{abstract}

Palavras-chave: comportamento ingestivo, estádio de lactação, ritmicidade, vacas leiteiras

\section{Ingestive Behavior Nycterohemeral Patterns of Dairy Cows, at the Beginning and at the End of Lactation, Fed a Corn Silage Based Diet}

\begin{abstract}
The effects of the stage of lactation upon the ingestive behavior nycterohemeral patterns were evaluated with eight dairy cows eating a corn silage based diet. Spectral analysis was performed through application of the finite Fourier transformation upon hourly duration of eating or ruminating time series. Variance analysis and inspection of phase diagrams and periodograms were done upon modified data. The mean meal duration and dry matter intake were larger while the unitary ruminating time was inferior at the beginning than at the end of the lactation. Nycterohemeral patterns of time spent eating or ruminating at the beginning and at the end of lactation followed mainly periodicities of, respectively, 24, 12, 8 and 4 hours and 24, 12 and 8 hours. A significant effect of stage of lactation was observed at the rhythm component 4 for both time spent eating and ruminating. Lactation stage affected some parameters of ingestive behavior and its rhythmicity patterns.
\end{abstract}

Key Words: dairy cows, ingestive behavior, rhythmicity, stage of lactation

\section{Introdução}

A ingestão de matéria seca é o principal fator que afeta a performance animal. $\mathrm{O}$ gado leiteiro pode modificar o comportamento de acordo com o tipo, quantidade e acessibilidade do alimento e práticas de manejo. O conhecimento do comportamento ingestivo do gado leiteiro pode ser utilizado pelos produtores, de modo a aumentar a produtividade e garantir a saúde e longevidade. Como exemplos práticos, poder-se-ia citar a localização de sistemas automáticos de fornecimento de água e alimento, a acessibilidade da ração, a redução da competição entre os animais por espaço, alimento e água, o horário e freqüência de distribuição da ração, entre outros (Albright, 1993).
Os ruminantes adaptam-se às diversas condições de alimentação, manejo e ambiente e modificam os parâmetros do comportamento ingestivo para alcançar e manter determinado nível de consumo, compatível com as exigências nutricionais (Hodgson, 1990). A mastigação dos alimentos provoca a redução do tamanho de partícula aquém do limite para que estas possam atravessar o orifício retículo-omasal, aumentando a densidade funcional, também crítica no escape de partículas do retículo-rúmen. A diminuição do tamanho de partículas ocorre tanto durante a ingestão quanto na ruminação, e a eficiência deste processo está diretamente ligada ao consumo de alimentos pelos ruminantes (Ulyatt et al., 1986).

O estudo do comportamento ingestivo e da ritmicidade pode auxiliar no entendimento dos fatores

\footnotetext{
${ }^{1}$ Faculdade de Agronomia, Universidade Federal de Pelotas, Bolsista do CNPq. Rua Fernandes Vieira, 181/601 - Porto Alegre, RS Brasil CEP: 90035-09. E.mail: vfried@ufpel.tche.br

${ }^{2}$ Faculdade de Ciências Agronômicas, Universidade Católica de Louvain, 1348, Bélgica (†2001).

${ }^{3}$ Faculdade de Ciências Agronômicas e do Ambiente, Universidade McGill, Québec, H9X 3V9. E.mail: dutilleul@musica.mcgill.ca

${ }^{4}$ Instituto Nacional de Alimentação Animal, Scheldeweg 68, B-9231 Melle-Gontrode, Bélgica.
} 
que induzem os animais a iniciar ou a terminar as refeições e, desta forma, o nível de consumo alcançado (Dulphy \& Faverdin, 1987).

Podem-se classificar os fatores, que afetam o comportamento ingestivo, como sendo ligados ao alimento, ao ambiente e ao animal. A composição químico-bromatológica, especialmente o teor de fibra em detergente neutro (FDN) e o tamanho de partícula são especialmente importantes para os ruminantes (Campbell et al., 1992). O horário, a freqüência e o intervalo de tempo entre os arraçoamentos influenciam a distribuição das atividades ingestivas (ingestão, ruminação e repouso) durante o dia (Deswysen et al., 1993), pois o fornecimento de ração induz o animal a ingerir (Chase et al., 1976; Jaster \& Murphy, 1983).

Os bovinos apresentam um padrão diurno de alimentação, tanto em pastejo (Forbes, 1986) como confinados (Ray \& Roubicek, 1971), ainda que o horário de distribuição do alimento e a quantidade fornecida possam influenciar o momento dos picos de ocorrência da atividade ingestiva (Chase et al., 1976; Jaster \& Murphy, 1983). As atividades ingestivas são concentradas em períodos discretos, distribuídos de forma não uniforme durante o nictêmero. Em geral, o número e a duração das refeições são mais variáveis que os períodos de ruminação (Dulphy \& Faverdin, 1987). Através do ajuste do número de refeições diárias e do seu tamanho (duração $\mathrm{x}$ taxa de ingestão), as vacas leiteiras podem ajustar o seu consumo num âmbito de curto prazo (Grant \& Albright, 1995).

Ainda que a definição do que constitui uma refeição (sua duração mínima e intervalo mínimo entre duas refeições consecutivas) varie entre os pesquisadores (Dulphy, 1971; Baumont, 1989), parece evidente que os padrões do comportamento ingestivo de vacas de alta produção e ao início da lactação devem ser diferentes dos de vacas com menor potencial produtivo e/ou ao final da lactação (Grant \& Albright, 1995).

O objetivo deste trabalho foi estudar algumas variáveis relacionadas ao comportamento ingestivo, e os padrões de distribuição, em vacas leiteiras com nível médio de produção, em dois estádios distintos: no início e no final da lactação.

\section{Material e Métodos}

Os experimentos foram conduzidos na estação experimental de Gontrode, em Gand, Bélgica (De

R. Bras. Zootec., v.31, n.5, p.2129-2138, 2002
Boever, 1991). No departamento de Nutrição Animal da Faculdade de Ciências Agronômicas da Universidade Católica de Louvain-la-Neuve, Bélgica, os dados gerados foram modificados pela transformação rápida de Fourier (TFF) e utilizados para avaliar a ritmicidade do comportamento ingestivo de vacas leiteiras, alimentadas com silagem de milho picada a 8 e a $4 \mathrm{~mm}$, nos experimentos 1 e 2 , respectivamente.

Foram utilizadas oito vacas Holandesas, na terceira lactação, por experimento. No primeiro experimento, as vacas produziram, no início e no final, respectivamente 31,2 e 19,7 litros de leite. No segundo experimento, as vacas produziram, no início e no final, respectivamente 32,9 e 20,1 litros de leite. Os animais foram ordenhados duas vezes ao dia. As pesagens foram realizadas pela manhã, antes do fornecimento da ração, após um jejum de 12 horas. No primeiro experimento, em média, as vacas pesaram, no início ( $10^{\mathrm{a}}$ semana) e no final (42a semana), respectivamente, 599 e $650 \mathrm{~kg}$. No segundo experimento, em média, as vacas pesaram, no início $\left(9^{\mathrm{a}}\right.$ semana) e no final ( $37^{\mathrm{a}}$ semana), respectivamente, 573 e $611 \mathrm{~kg}$. Os animais foram adaptados à dieta por 10 dias e aos arreios por três dias. Foi utilizado o alojamento individual tipo "tie-stall", com piso de borracha. A dieta foi distribuída duas vezes ao dia, às $8 \mathrm{~h} 30$ e $16 \mathrm{~h}$, e, em cada arraçoamento, esta foi composta por silagem de milho fornecida à vontade, além de um $\mathrm{kg}$ de farelo de soja (matéria fresca) + $100 \mathrm{~g}$ de premix mineral e vitamínico. No experimento $1, o$ milho para silagem foi colhido no ponto de grão leitoso, picado em pedaços de $8 \mathrm{~mm}$ e apresentou $27,8 \%$ de fibra bruta. No experimento 2, o milho para silagem foi colhido no ponto de grão firme, picado em pedaços de $4 \mathrm{~mm}$ e apresentou $22,8 \%$ de fibra bruta. O consumo voluntário foi obtido pela diferença entre a quantidade de alimento fornecido e as sobras. Os valores mostrados na Tabela 1 referem-se à média do consumo medido no estádio inicial (respectivamente $10^{\mathrm{a}}$ e $9^{\mathrm{a}}$ semanas) e no estádio final (respectivamente $42^{\mathrm{a}}$ e $37^{\mathrm{a}}$ semanas) da lactação, nos experimentos 1 e 2 .

Nos experimentos 1 e 2 , os tratamentos constituíram-se do estádio inicial $\left(10^{\mathrm{a}}\right.$ e $9^{\mathrm{a}}$ semanas, respectivamente) e do estádio final (respectivamente $42^{\mathrm{a}} \mathrm{e}$ $37^{\mathrm{a}}$ semanas) da lactação.

O comportamento ingestivo foi registrado pela percepção dos movimentos mandibulares, através de um tubo flexível de silicone fixado por arreios sob a mandíbula. Os movimentos mandibulares comprimi- 
ram o tubo de silicone, e as mudanças na pressão do ar contido neste tubo foram enviadas a um transdutor (tipo 4 AI-50/020/56 da Jumo) e transformadas em sinal elétrico. Esses sinais foram registrados por um equipamento de quatro canais (fisiógrafo do tipo MC.6850-4L da Ankersmit). A taxa de passagem do papel foi de $1 \mathrm{~cm}$ por minuto.

O comportamento ingestivo foi classificado em três atividades principais: ingestão, ruminação e descanso. O tempo de ingestão incluiu a apreensão e manipulação do alimento, mastigação e deglutição do bolo alimentar. O tempo de ruminação incluiu regurgitação, remastigação e redeglutição do bolo, assim como o interciclo. O tempo de descanso compreendeu também a ingestão de líquidos. Foram adotados os seguintes critérios para a classificação das atividades do comportamento ingestivo: uma refeição ou um período de ruminação deveria durar no mínimo sete minutos e atividades alimentares ou ruminativas espaçadas de menos de sete minutos foram consideradas como pertencentes, respectivamente, à mesma refeição ou período de ruminação (Dulphy, 1971).

Em cada experimento, os animais e os tratamentos foram distribuídos ao acaso, segundo um delineamento experimental de duplo quadrado latino (oito vacas $\mathrm{x}$ dois estádios de lactação $\mathrm{x}$ dois períodos). As variáveis relacionadas ao comportamento ingestivo (duração e número das atividades e consumo) foram estudados através da análise de variância, que considerou os efeitos de quadrado latino $(\mathrm{GL}=1)$, estádio da lactação $(\mathrm{GL}=1)$, período aninhado dentro de cada quadrado latino $(\mathrm{GL}=2)$ e animal aninhado dentro de cada quadrado latino $(\mathrm{GL}=6)$. O teste de médias usado foi o Student-Neuman-Keuls. Onível mínimo de significância para rejeição da hipótese de nulidade foi de 0,05.

A decomposição espectral das séries temporais de 24 valores dos tempos gastos ingerindo e ruminando em um número inteiro de ciclos a cada $24 \mathrm{~h}$, referidos como componente de ritmo, foi realizada pela Transformação Finita de Fourier (TFF) e da análise de variância e periodograma, avaliando os efeitos estádios de lactação, período hierarquizado em cada quadrado latino, animais hierarquizados em cada quadrado latino, e a interação entre estádio de lactação $\mathrm{x}$ animais $\mathrm{x}$ período hierarquizado em cada quadrado latino, em cada freqüência considerada.

A análise estatística da TFF foi realizada dentro dos mesmos princípios descritos por Deswysen et al. $(1989 ; 1993)$ e executada usando os procedimentos

R. Bras. Zootec., v.31, n.5, p.2129-2138, 2002
GLM do SAS (1989) e SPECTRA do SAS (1988a). As hipóteses de nulidade testadas foram rejeitadas quando o valor de probabilidade foi menor que 0,05 . A atribuição das causas das diferenças entre os níveis das variáveis classificatórias (estádio da lactação e animal) detectadas pela análise de variância da TFF, se devido à diferença de amplitude ou defasagem no tempo de ocorrência dos picos de atividade (ingestão ou ruminação) em relação ao início dos registros do comportamento, foi realizada por meio da inspeção dos valores das ordenadas de periodogramas e diagramas de fase. A importância relativa dos componentes do ritmo em relação à dispersão total das séries temporais foi calculada como uma proporção entre o seu valor do periodograma e o valor total do periodograma (a soma dos valores de todos os componentes de ritmo), e foram relacionados ao consumo voluntário, usando o coeficiente de correlação de Pearson, calculado pelo procedimento CORR do SAS (1988b). No presente trabalho, somente serão abordados os resultados relativos ao efeito estádio da lactação.

\section{Resultados e Discussão}

Os valores de consumo de matéria seca e das variáveis relacionadas ao comportamento ingestivo são apresentados na Tabela 1. Em ambos os experimentos, o consumo médio de matéria seca diminuiu no final da lactação em comparação com o início (Tabela 1), o que estaria relacionado às menores necessidades nutricionais no período final, em decorrência da menor produção de leite (Dado \& Allen, 1994; NRC, 2001).

Observa-se, na Tabela 1, que, embora o consumo fosse maior durante a fase inicial de lactação, não foram detectadas diferenças significativas quanto à duração total diária de ingestão e número de refeições entre os estádios inicial e final da lactação. No primeiro experimento, também não foi detectada diferença quanto à duração média das refeições. Provavelmente a diferença no consumo voluntário de aproximadamente $1,4 \mathrm{~kg}$ de matéria seca ou de 0,38 unidades percentuais não foi suficiente para afetar essas características do comportamento dos animais. Isto significa que, ao início da lactação, os animais consumiram mais rapidamente o alimento em relação ao final da mesma. Coulon et al. (1987) verificaram que as vacas aumentaram o consumo, ao início da lactação, através do aumento do tempo dedicado à 
Tabela 1 - Efeitos do estádio de lactação sobre as variáveis relacionadas ao comportamento ingestivo (média dos dois períodos)

Table 1 - Effects of lactation stage upon the variables related to the ingestive behavior (mean of the two periods)

\begin{tabular}{|c|c|c|c|c|}
\hline \multirow[b]{3}{*}{$\begin{array}{l}\text { Variáveis } \\
\text { Variables }\end{array}$} & \multicolumn{2}{|c|}{$\begin{array}{l}\text { Experimento } 1(8 \mathrm{~mm}) \\
\text { Experiment } 1(8 \mathrm{~mm})\end{array}$} & \multicolumn{2}{|c|}{$\begin{array}{l}\text { Experimento } 2(4 \mathrm{~mm}) \\
\text { Experiment } 2(4 \mathrm{~mm})\end{array}$} \\
\hline & \multicolumn{4}{|c|}{$\begin{array}{l}\text { Estádio de lactação } \\
\text { Lactation stage }\end{array}$} \\
\hline & $\begin{array}{c}\text { Início } \\
\text { Beginning }\end{array}$ & $\begin{array}{l}\text { Final } \\
\text { End }\end{array}$ & $\begin{array}{c}\text { Início } \\
\text { Beginning }\end{array}$ & $\begin{array}{c}\text { Final } \\
\text { End }\end{array}$ \\
\hline $\begin{array}{l}\text { Consumo }(\mathrm{kg} \mathrm{MS} / \mathrm{d}) \\
\text { Intake }(k g D M / d)\end{array}$ & 12,4 & $11,0 *$ & 13,0 & $11,6 *$ \\
\hline $\begin{array}{l}\text { Consumo }(\% \mathrm{PV} / \mathrm{d}) \\
\text { Intake }(\% L W / d) \\
\text { Tempo de ingestão } \\
\text { Eating time }\end{array}$ & 2,07 & $1,69 *$ & 2,27 & $1,90 *$ \\
\hline $\begin{array}{l}\text { Min/d } \\
\operatorname{Min} / d\end{array}$ & 292,1 & $283,4 \mathrm{~ns}$ & 272,6 & $253,8 \mathrm{~ns}$ \\
\hline $\begin{array}{l}\text { Min/kg MS /d } \\
M i n / k g D M / d\end{array}$ & 23,6 & $25,8 \mathrm{~ns}$ & 21,0 & $21,9 \mathrm{~ns}$ \\
\hline $\begin{array}{l}\text { Número de refeições } \\
\text { Number of meals }\end{array}$ & 8,6 & $5,4 \mathrm{~ns}$ & 8,0 & $9,0 \mathrm{~ns}$ \\
\hline $\begin{array}{l}\text { Duração das refeições (min) } \\
\text { Duration of meals }(\min ) \\
\text { Tempo de ruminação }\end{array}$ & 34,0 & $33,7 \mathrm{~ns}$ & 34,1 & $28,2 *$ \\
\hline $\begin{array}{l}\text { Ruminating time } \\
\text { Min/d } \\
\text { Min/d }\end{array}$ & 522,7 & $519,0 \mathrm{~ns}$ & 468,0 & $442,0 \mathrm{~ns}$ \\
\hline $\begin{array}{l}\mathrm{Min} / \mathrm{kg} \mathrm{MS} / \mathrm{d} \\
\mathrm{Min} / \mathrm{kg} \mathrm{DM} / d\end{array}$ & 42,2 & $47,3 *$ & 36,0 & $38,1 \mathrm{~ns}$ \\
\hline $\begin{array}{l}\text { Número de períodos de ruminação } \\
\text { Number of rumination periods }\end{array}$ & 14,1 & $13,9 \mathrm{~ns}$ & 12,8 & $12,7 \mathrm{~ns}$ \\
\hline $\begin{array}{l}\text { Duração períodos de ruminação (min) } \\
\text { Duration of rumination periods (min) }\end{array}$ & 37,1 & $37,4 \mathrm{~ns}$ & 36,7 & $34,8 \mathrm{~ns}$ \\
\hline $\begin{array}{l}\text { Número de bolos de ruminação } \\
\text { Number of rumination boli }\end{array}$ & 519,0 & $498,0 \mathrm{~ns}$ & 450,0 & $420,0 \mathrm{~ns}$ \\
\hline $\begin{array}{l}\text { Duração dos bolos de ruminação (s) } \\
\text { Rumination boli duration }(s)\end{array}$ & 60,5 & $62,7 *$ & 62,4 & $63,1 \mathrm{~ns}$ \\
\hline
\end{tabular}

Médias em cada linha, em cada experimento, seguidas por * e por ns são, respectivamente, significativa e não significativamente diferentes $(P<0,05)$, segundo o teste de Student-Neuman-Keuls.

Means within a row for each experiment, followed by * or ns are significantly or not significantly different $(P<0.05)$, accordingly to Student-Neuman-Keuls test.

mastigação, quando este era inicialmente pequeno ou aumentando a taxa de movimentos mastigatórios. Segundo os mesmos autores, raramente as vacas aumentaram ambas as variáveis na mesma oportunidade. Estes autores também verificaram uma alta correlação entre potencial de produção de leite e taxa de ingestão de alimentos.

Costa (2000) estudou o comportamento de vacas Jersey aos 30, 60 e 90 dias após o parto. Constatou que o período da curva de lactação influenciou o comportamento ingestivo dos animais, sendo que o tempo médio de ingestão foi superior aos 30 e 60 dias pós parto (371 e $356 \mathrm{~min} / \mathrm{dia}$ ) em relação aos 90 dias pós parto (325 $\mathrm{min} / \mathrm{dia})$.
No experimento 2, observou-se a diminuição da duração média das refeições, quando as vacas passaram do estádio inicial para o final (Tabela 1). Isto concorda com Grant \& Albright (1995), que, num trabalho de revisão, concluíram que vacas de alta produção aumentam o seu consumo através do incremento da duração das refeições e da redução dos tempos de ingestão e de ruminação por unidade de matéria seca consumida. Este fato, além da semelhança dos tempos de ingestão entre os dois estádios de lactação, confirma os resultados de diversos autores de que o aumento das exigências nutricionais e, conseqüentemente, do consumo promove o aumento da taxa de ingestão ( $\mathrm{kg}$ de matéria seca/minuto), 
podendo alterar ou não o tempo total de ingestão em bovinos de leite (Coulon et al., 1987; Deswysen et al., 1987; Albright, 1993; Grant \& Albright, 1995), ovinos (Fischer, 1996) e bovinos de corte (Fischer, 1996).

O tempo diário total de ruminação não variou entre os estádios de lactação (Tabela 1), o que concorda com os resultados obtidos por Costa (2000). A duração total da ruminação não aumenta na mesma proporção que o consumo e, em consequiência, as durações unitárias de ruminação e de mastigação da matéria seca ou da fibra em detergente neutro (FDN) (minutos de atividade/kg de matéria seca ou FDN) diminuem à medida que o nível de ingestão aumenta (Welch \& Smith, 1969; Deswysen et al., 1987; Fischer, 1996). Entre as causas possíveis da redução das durações unitárias de ruminação e mastigação podem-se citar o aumento da eficiência do processo de trânsito das partículas da digesta do retículo-rúmen para o omaso ou um aumento do tamanho crítico de passagem das partículas pelo orifício retículo-omasal, como foi sugerido por Deswysen et al. (1987), ou maior eficiência na redução de partículas por movimento mandibular (Bae et al., 1981; De Boever et al., 1990).

As vacas consumindo silagem picada a $8 \mathrm{~mm}$, no início da lactação, ruminaram mais rapidamente que ao final da lactação, como pode ser observado pelo menor índice de ruminação, expresso como minutos por kg de matéria seca consumida (Tabela 1), o que está de acordo com os resultados de Dado \& Allen (1994), sugerindo maior eficiência da redução do tamanho de partículas ou a passagem de partículas de maior tamanho pelo orifício retículo-omasal (Deswysen et al., 1987). Shaver et al. (1985) observaram que vacas secas apresentaram maior índice de ruminação e de mastigação ( $\mathrm{min} / \mathrm{kg}$ MS consumida) em relação ao início da lactação.

Embora os experimentos 1 e 2 não tenham sido comparados estatisticamente, pode-se observar que a maioria das variáveis relacionadas ao comportamento ingestivo apresentou maiores valores numéricos quando as vacas consumiram a silagem picada a $8 \mathrm{~mm}$, comparados com a silagem picada a $4 \mathrm{~mm}$. Este fato foi, provavelmente, devido ao maior teor de fibra bruta da silagem picada a $8 \mathrm{~mm}$ e ao maior tamanho de partícula. O teor de fibra foi positivamente relacionado ao tempo de ruminação (Welch \& Smith, 1970; Dulphy et al., 1980) e aos tempos total e unitário de ingestão (Beauchemin \& BuchananSmith, 1989). O tamanho de partícula foi positiva- mente relacionado com a duração do tempo de ruminação e de mastigação (Welch \& Smith, 1971).

As distribuições dos tempos horários de ingestão e de ruminação, nos estádios inicial (B) e final (E) dos experimentos 1 e 2, são apresentadas, respectivamente, nas Figuras 1 e 2. Os resultados da análise de variância das médias diárias dos tempos de ingestão e de ruminação (componente do ritmo $=0$ ) e dos componentes de ritmo 1 a 12 dos experimentos 1 e 2 são apresentados, respectivamente, nas tabelas 2 e 3 . Os periodogramas dos tempos de ingestão e de ruminação dos experimentos 1 e 2 são apresentados, respectivamente, nas figuras 3 e 4 . Um limite mínimo de importância foi calculado como igual a $10 \%$ do valor total da dispersão das séries dos tempos gastos com a ingestão e a ruminação, calculada como a média entre tratamentos, e indicada como um traço descontínuo nas Figuras 3 e 4.

A atividade de ingestão foi concentrada entre $6 \mathrm{e}$ $19 \mathrm{~h}$, atingindo os valores máximos entre 9 e $10 \mathrm{~h} \mathrm{e}$ entre 17 e 18h. Além destes picos de atividade ingestiva coincidentes com o horário de distribuição da ração, as vacas exibiram um terceiro pico entre 5 e $7 \mathrm{~h}$, embora de magnitude bem inferior. As vacas que receberam silagem picada a $8 \mathrm{~mm}$ apresentaram maior atividade noturna de ingestão, entre 20 e 01 horas em relação às que receberam silagem picada a $4 \mathrm{~mm}$ (Figura 1).

A concentração da atividade ingestiva durante a fase diurna do dia também foi observada em novilhas leiteiras (Deswysen et al., 1989), novilhos confinados (Ray \& Roubicek, 1971) e ruminantes sob pastejo (Forbes, 1986; Fischer, 1996).

A atividade de ruminação foi mais consistente entre 20 e $5 \mathrm{~h}$, embora os animais tenham apresentado uma expressiva atividade de ruminação, respectivamente nos experimentos 1 e 2 , entre 10 e $16 \mathrm{~h}$ e entre 11 e 16h (Figura 2). Estes resultados estão de acordo com Deswysen et al. $(1989,1993)$, trabalhando com novilhas Holandesas, que constataram atividade de ruminação mais consistente durante a madrugada, mas também a presença de atividade de ruminação significativa entre os dois arraçoamentos. As vacas que receberam silagem picada a $8 \mathrm{~mm}$ apresentaram maior atividade horária de ruminação em relação às que receberam silagem picada a $4 \mathrm{~mm}$ (Figura 2).

Os resultados da análise de variância da TFF das séries de 24 horas dos tempos de ingestão e de ruminação, no experimento 1 , indicam um efeito significativo do estádio de lactação para o tempo

\section{R. Bras. Zootec., v.31, n.5, p.2129-2138, 2002}




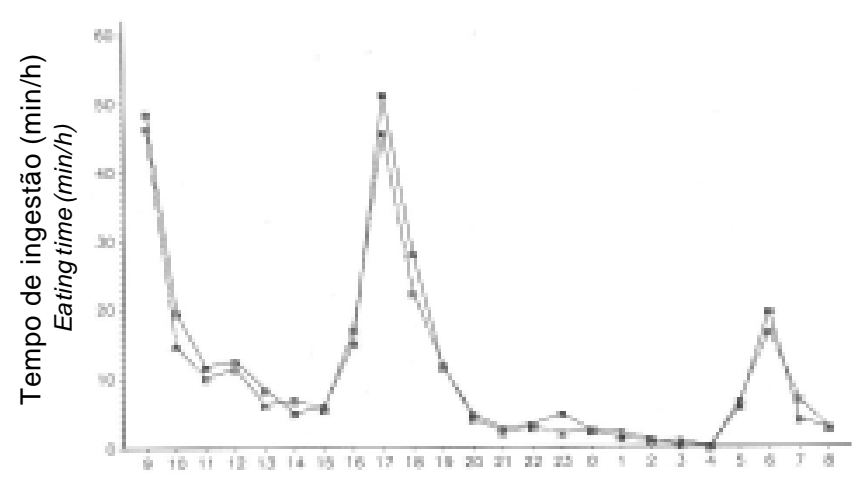

Horas do dia

Hours of the day

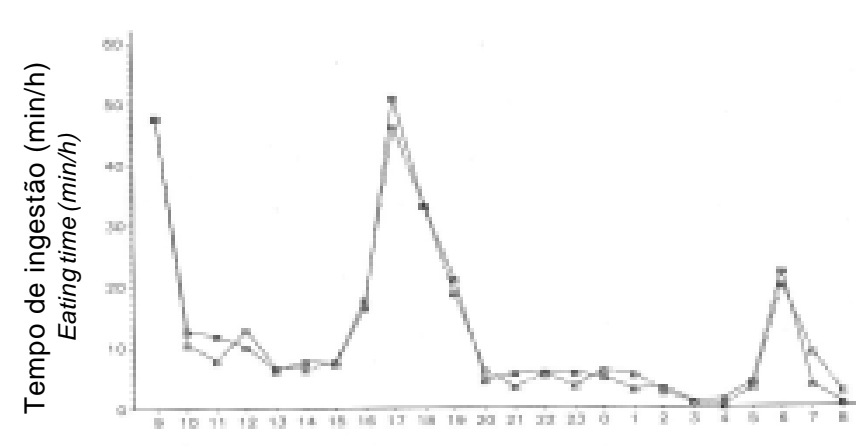

Horas do dia

Hours of the day

Figura 1 - Duração horária do tempo de ingestão (minutos/hora) de vacas alimentadas com silagem de milho picada a $4 \mathrm{~mm}$ (no alto) e $8 \mathrm{~mm}$ (em baixo) ao início (B) e ao final (E) da lactação.

Figure 1 - Hourly duration of eating time (minutes/hour) of cows fed corn silage cut into $4 \mathrm{~mm}$ (above) and 8 $\mathrm{mm}$ (below) at the beginning $(B)$ and end $(E)$ of lactation.

gasto ingerindo e ruminando no componente de ritmo 4 (Tabela 2).

A frequiência 1 define um dos componentes fundamentais do ritmo nictemeral, e é representado por uma função trigonométrica com ciclos a cada 24 horas, e a frequiência 4 , harmônica em relação à freqüência 1, corresponde a ciclos de 6 horas (Tabela 2). A freqüência 0 corresponde à média horária dos tempos gastos ingerindo ou ruminando.

Em relação ao experimento 2, constatou-se um efeito significativo do estádio de lactação para o tempo gasto ingerindo e ruminando, respectivamente nos componentes de ritmo 0,1 e 4 (Tabela 3).

As diferenças entre os estádios de lactação quan-
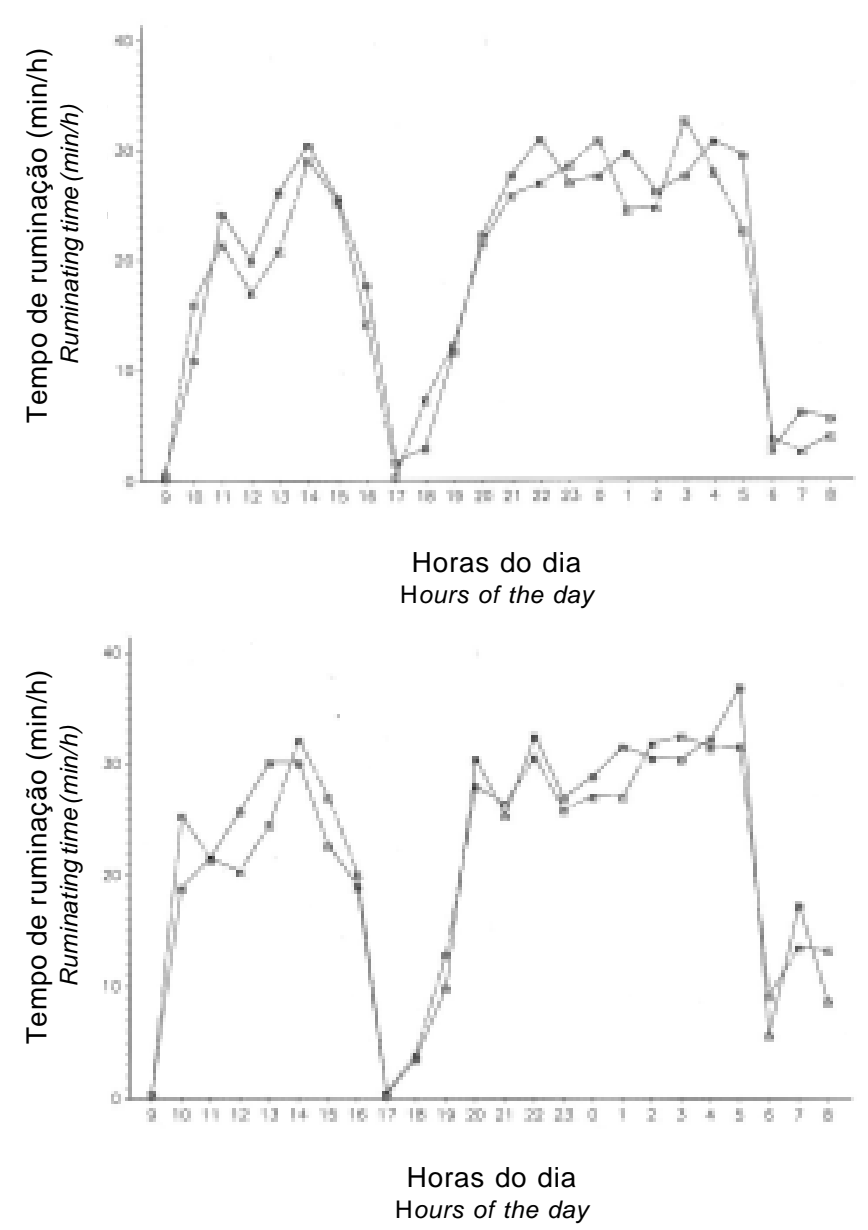

Figura 2 - Duração horária do tempo de ruminação (minutos/hora) de vacas alimentadas com silagem de milho picada a $4 \mathrm{~mm}$ (no alto) e 8 $\mathrm{mm}$ (em baixo) ao início (B) e ao final (E) da lactação.

Figure 2 - Hourly duration of ruminating time (minutes/hour) of cows fed corn silage cut into $4 \mathrm{~mm}$ (above) and $8 \mathrm{~mm}$ (below) at the beginning $(B)$ and end $(E)$ of lactation.

to ao padrão de dispersão da atividade de ingestão ou ruminação foram detectadas principalmente na amplitude, ou seja, entre as ordenadas do periodograma, nos dois experimentos (Figuras 3 e 4 ).

Não houve diferenças entre os estádios de lactação quanto aos valores do diagrama de fase, significando que estes não influenciaram o momento de ocorrência dos valores máximos para os tempos horários de ingestão ou ruminação. Este fato deve-se, provavelmente, à grande influência dos horários de arraçoamento sobre a ocorrência dos picos de atividade, tanto de ingestão como de ruminação.

No experimento 1, as análises de variância da 
Tabela 2 - Análise de variância da média horária do tempo gasto ingerindo e ruminando (minutos/h) e da transformação finita de Fourier para cada componente de ritmo, no experimento 1 (8 $\mathrm{mm})$

Table 2 - Variance analysis of eating and ruminating time (minutes/h) hourly mean and of the finite Fourier transform for each rhythm component in experiment $1(8 \mathrm{~mm})$

\begin{tabular}{lcccc}
\hline & \multicolumn{2}{c}{$\begin{array}{c}\text { Tempo de ingestão } \\
\text { Eating time }\end{array}$} & \multicolumn{2}{c}{$\begin{array}{c}\text { Tempo de ruminação } \\
\text { Ruminating time }\end{array}$} \\
\cline { 2 - 4 } $\mathrm{N}^{\circ}$ de ciclos/24h & \multicolumn{2}{c}{$\begin{array}{c}\text { Vacas } \\
\text { Number of cycles/24h } h\end{array}$} & $\begin{array}{c}\text { Estádio de lactação } \\
\text { Lactation stage }\end{array}$ & $\begin{array}{c}\text { Vacas } \\
\text { Cows }\end{array}$ \\
\hline $0^{\mathrm{a}}$ & 0,1184 & 0,1228 & 0,7638 & 0,0001 \\
1 & 0,1668 & 0,0007 & 0,1576 & 0,0068 \\
2 & 0,9134 & 0,0001 & 0,1348 & 0,0001 \\
3 & 0,1981 & 0,0001 & 0,0516 & 0,0241 \\
4 & 0,0099 & 0,0183 & 0,0191 & 0,1751 \\
5 & 0,7817 & 0,5970 & 0,6737 & 0,5982 \\
6 & 0,3162 & 0,0677 & 0,1451 & 0,4113 \\
7 & 0,7721 & 0,4705 & 0,9077 & 0,4088 \\
8 & 0,2031 & 0,4629 & 0,7938 & 0,7974 \\
9 & 0,3571 & 0,4288 & 0,6471 & 0,1305 \\
10 & 0,7740 & 0,1157 & 0,9540 & 0,2827 \\
12 & 0,0714 & 0,3162 & 0,0732 & 0,8560 \\
\hline
\end{tabular}

a Média diária da proporção horária do tempo gasto ingerindo ou ruminando.

a Daily mean of hourly proportions of eating or ruminating time.

Tabela 3 - Análise da variância da média horária do tempo gasto ingerindo e ruminando (minutos/h) e da transformação finita de Fourier para cada componente de ritmo, no experimento $2(4 \mathrm{~mm})$

Table 3 - Variance analysis of eating and ruminating time (minutes/h) hourly mean and of the finite Fourier transform for each rhythm component in experiment $2(4 \mathrm{~mm})$

\begin{tabular}{|c|c|c|c|c|}
\hline \multirow[b]{2}{*}{$\begin{array}{l}\mathrm{N}^{\circ} \text { de ciclos } / 24 \mathrm{~h} \\
\text { Number of cycles } / 24 \mathrm{~h}\end{array}$} & \multicolumn{2}{|c|}{$\begin{array}{c}\text { Tempo de ingestão } \\
\text { Eating time }\end{array}$} & \multicolumn{2}{|c|}{$\begin{array}{c}\text { Tempo de ruminação } \\
\text { Ruminating time }\end{array}$} \\
\hline & $\begin{array}{c}\text { Estádio de lactação } \\
\text { Lactation stage }\end{array}$ & $\begin{array}{l}\text { Vacas } \\
\text { Cows }\end{array}$ & $\begin{array}{c}\text { Estádio de lactação } \\
\text { Lactation stage }\end{array}$ & $\begin{array}{l}\text { Vacas } \\
\text { Cows }\end{array}$ \\
\hline$\overline{0^{\mathrm{a}}}$ & 0,0001 & 0,0001 & 0,0048 & 0,0001 \\
\hline 1 & 0,0239 & 0,0001 & 0,6149 & 0,0001 \\
\hline 2 & 0,2444 & 0,0001 & 0,6102 & 0,0001 \\
\hline 3 & 0,0799 & 0,0001 & 0,0958 & 0,3116 \\
\hline 4 & 0,0172 & 0,0057 & 0,8994 & 0,0348 \\
\hline 5 & 0,4893 & 0,0138 & 0,4405 & 0,3201 \\
\hline 6 & 0,2546 & 0,0001 & 0,1023 & 0,0009 \\
\hline 7 & 0,3946 & 0,0025 & 0,1189 & 0,0168 \\
\hline 8 & 0,3305 & 0,0022 & 0,1158 & 0,0908 \\
\hline 9 & 0,3858 & 0,0102 & 0,8546 & 0,0373 \\
\hline 10 & 0,1460 & 0,3444 & 0,8772 & 0,8367 \\
\hline 11 & 0,3636 & 0,0076 & 0,7073 & 0,6205 \\
\hline 12 & 0,9602 & 0,0657 & 0,4485 & 0,7327 \\
\hline
\end{tabular}

a Média diária da proporção horária do tempo gasto ingerindo ou ruminando.

Daily mean of hourly proportions of eating or ruminating time.

TFF e do periodograma mostraram que, no início da lactação, a distribuição nictemeral do tempo gasto ingerindo na freqüência de 4 ciclos/24 h apresentou maior amplitude que ao final da lactação. Entretanto, no experimento 2 , no início da lactação, a distribuição nictemeral do tempo gasto ingerindo, nas freqüências de 1 e 4 ciclos $/ 24 \mathrm{~h}$, apresentou maior amplitude que ao final da lactação (Figura 3). Estes resultados provavelmente estão relacionados à evolução do tempo gasto ingerindo por hora. Durante os três picos de atividade ingestiva (Figura 1), as vacas, no início da lactação, apresentaram maior tempo de ingestão por hora que aquelas ao final da lactação, enquanto que, durante os demais horários, a diferença entre o 
tempo de ingestão das vacas ao início e final da lactação foi bem menor, o que gerou as diferenças de amplitude. Este fato pode evidenciar a importância de se manter alimento no cocho à disposição das vacas durante todo o nictêmero, especialmente àquelas no início da lactação, para não restringir o consumo.

Nos dois experimentos, a dispersão do padrão nictemeral do tempo de ingestão nos estádios inicial e final da lactação foi descrita, principalmente, pelos componentes de ritmo 1, 2, 3 e 6, correspondentes, respectivamente, a ciclos a cada $24,12,8$ e 4 horas (Figura 3). A importância relativa do tempo de ingestão, associado com os componentes de ritmo 1 , 2, 3 e 6, foi calculada para cada estádio da lactação como porcentagem da dispersão total explicada por estes componentes do ritmo.

No experimento 1, o componente de ritmo 1 descreveu 16,69 e $16,76 \%$ da dispersão total das proporções de tempo gasto ingerindo, respectivamente, nos estádios inicial e final da lactação, o componente de ritmo $2,14,42$ e $15,58 \%$, o componente de ritmo 3, 19,43 e $21,51 \%$ e o componente $6,12,44$ e $11,11 \%$, enquanto, no experimento 2 , o componente de ritmo 1 descreveu 21,07 e 19,86\%, o componente de ritmo 2 , 12,51 e $12,24 \%$, o componente de ritmo $3,21,55$ e $16,64 \%$ e o componente $6,15,83$ e 13,61\% (Figura 3).

A importância dos componentes de ritmo 1 e 2 foi parcialmente promovida pela concentração da atividade de ingestão durante o período diurno. A importância do componente de ritmo 3 pode ser relacionada ao intervalo de aproximadamente 8 horas entre os arraçoamentos da manhã e da tarde, enquanto a importância relativa do componente de ritmo 6 pode estar relacionado ao intervalo de tempo decorrido entre os picos de atividade ingestiva, de aproximadamente 4 e 8 horas. Deswysen et al. $(1989,1993)$ mostraram que o horário de arraçoamento e o tempo decorrido entre refeições principais influenciaram a importância dos diferentes componentes de ritmo. A ocorrência de refeições intermediárias pode exercer um efeito benéfico sobre o consumo, ao atenuar as flutuações do $\mathrm{pH}$ e da produção de metabólitos ruminais (Deswysen et al., 1993).

As diferenças entre os estádios de lactação, quanto ao padrão de dispersão da atividade de rumi- nação, foram detectadas, principalmente na amplitude, para o componente de ritmo 4 , no experimento 1 (Figura 4).

A análise do periodograma mostrou que, ao final da lactação, a distribuição nictemeral do tempo gasto ruminando apresentou maior amplitude que no início da lactação, na freqüência de 4 ciclos $/ 24 \mathrm{~h}$, provavelmente refletindo a menor consistência da atividade, com maior número de pequenos picos da atividade de ruminação (Figura 4).

Em ambos os experimentos, o padrão nictemeral do tempo gasto ruminando foi descrito principalmente pelos componentes de ritmo 1,2 e 3 . No experimento 1 , o componente de ritmo 1 descreveu 15,15 e $13,71 \%$ da dispersão das proporções de tempo gasto ruminando, respectivamente, nos estádios inicial e final da lactação, o componente de ritmo $2,36,05$ e $33,53 \%$ e o componente de ritmo $3,25,32$ e $19,99 \%$, enquanto, no experimento 2 , o componente de ritmo 1 descreveu 15,95 e $20,97 \%$, o componente de ritmo $2,45,22$ e $45,43 \%$ e o componente de ritmo 3, 21,49 e 14,76\% (Figura 4).

A importância do componente de ritmo $1 \mathrm{em}$ relação aos outros componentes de ritmo, para descrever o padrão de distribuição circadiano da ruminação, no início e no final da lactação, está de acordo com observações anteriores sobre consistência da ruminação durante o período noturno (Gordon, 1958; Gordon \& Mc Allister, 1970; Deswysen et al., 1989, 1993). A importância dos componentes de ritmo 2 e 3 foi associada, respectivamente, à atividade de ruminação no início da tarde, também descrita por Pearce (1965) e Deswysen et al. $(1989,1993)$ e à existência de um pequeno pico da atividade entre 7 e $8 \mathrm{~h}$.

Em ambos os experimentos, pode-se verificar que os valores de amplitude para o tempo gasto ingerindo foram superiores aos do tempo de ruminação, exceto no componente de ritmo 2. A proporção do tempo gasto ingerindo foi mais variável, durante o nictêmero, do que o tempo para ruminação. Estes resultados estão de acordo com aqueles obtidos por Deswysen et al. (1993) e na revisão de literatura de Dulphy \& Faverdin (1987), que caracterizaram a atividade de ruminação como composta por períodos com duração mais homogênea e de distribuição mais uniforme durante o dia. 


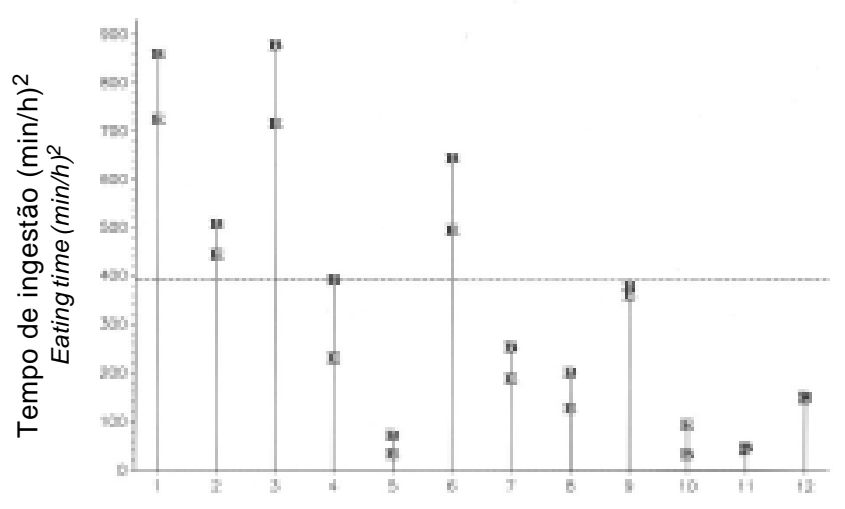

Número de ciclos/24 horas Number of cycles/24 hours

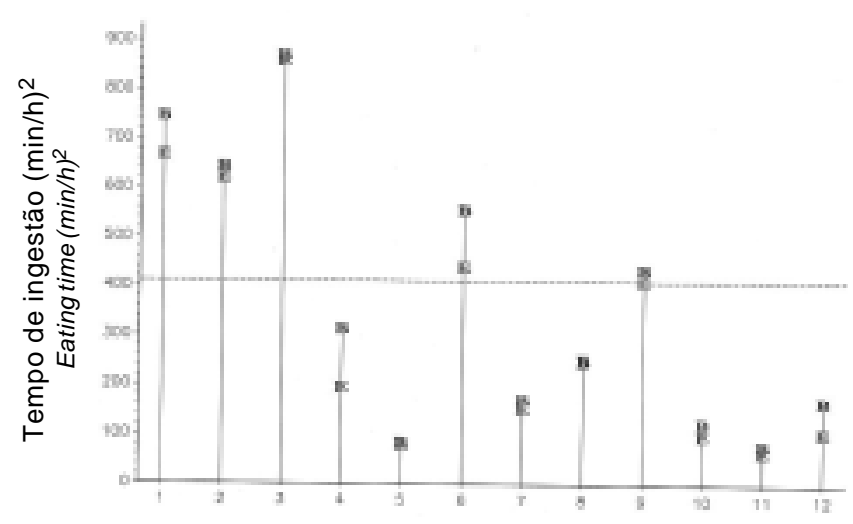

Número de ciclos/24 horas Number of cycles/24 hours

Figura 3 - Periodograma do tempo de ingestão (minutos/hora) ${ }^{2}$ de vacas alimentadas com silagem de milho picada a $4 \mathrm{~mm}$ (no alto) e $8 \mathrm{~mm}$ (em baixo) ao início (B) e ao final (E) da lactação.

Figure 3 - Periodogram of eating time (minutes/hour) ${ }^{2}$ of cows fed corn silage cut into $4 \mathrm{~mm}$ (above) and 8 $\mathrm{mm}$ (below) at the beginning $(B)$ and end $(E)$ of lactation.

\section{Conclusões}

A distribuição nictemeral do comportamento ingestivo de vacas leiteiras em produção foi influenciada pelo estádio de lactação, provavelmente devido às diferentes necessidades nutricionais e, conseqüentemente, a diferentes consumos voluntários.

\section{Literatura Citada}

ALBRIGHT, J.L. Nutrition, feeding and calves. Feeding behavior of dairy cattle. Journal of Dairy Science, v.76, n.2, p.485-498, 1993.

R. Bras. Zootec., v.31, n.5, p.2129-2138, 2002
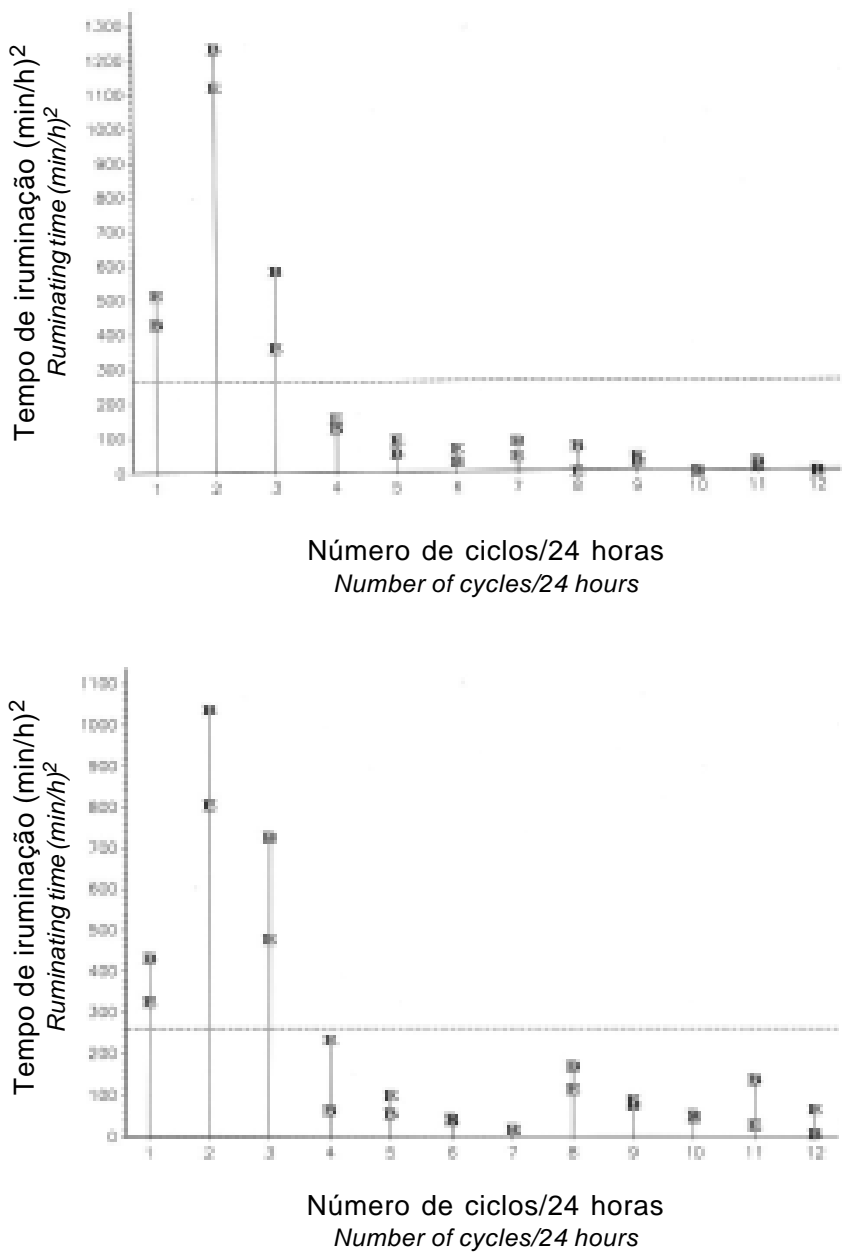

Figura 4 - Periodograma do tempo de ruminação (minutos/hora) ${ }^{2}$ de vacas alimentadas com silagem de milho picada a $4 \mathrm{~mm}$ (no alto) e s8 $\mathrm{mm}$ (em baixo) ao início (B) e ao final (E) da lactação.

Figure 4 - Periodogram of ruminating time (minutes/hour) ${ }^{2}$ of cows fed corn silage cut into $4 \mathrm{~mm}$ (above) and $8 \mathrm{~mm}$ (below) at the beginning $(B)$ and end $(E)$ of lactation.

BAE, D.H.; WELCH, J.G.; SMITH, A.M. Efficiency of mastication in relation to hay intake by cattle. Journal of Animal Science, v.52, n.6, p.1371-1375, 1981.

BAUMONT, R. Etat de repletion du reticulo-rumen et ingestion de fourrages : incidences sur le contrôle à court term de la quantité de foin ingerée par le mouton. Clermont-Ferrand: Institute des Recherches Agricoles, 1989. 159p. Tese (Doutorado em Zootecnia) - Institute des Recherches Agricoles, 1989.

BEAUCHEMIN, K.A.; BUCHANAN-SMITH, J.G. Effects of dietary neutral detergent fiber concentration and supplementary long hay on chewing activities and milk production of dairy cows. Journal of Dairy Science, v.72, p.2288-2300, 1989.

CAMPBELL, C.P.; MARSHALL, S.A.; MANDELL, I.B. et al. Effects of source of dietary neutral detergent fiber on chewing 
behavior in beef cattle fed pelleted concentrates with or without supplemental roughage. Journal of Animal Science, v.70, n.7, p.894-903, 1992.

CHASE, L.E.; WANGSNESS, P.J.; BAUMGARDT, B.R. Feeding behavior of steers fed a complete mixed ration. Journal of Dairy Science, v.59, n.11, p.1923-1928, 1976.

COSTA, C.O. Comportamento sexual e ingestivo de vacas Jersey confinadas, durante a fase inicial da lactação. Pelotas: Universidade Federal de Pelotas, 2000. 154p. Dissertação (Mestrado) - Universidade Federal de Pelotas, 2000.

COULON, J.B.; DOREAU, M.; REMOND, B. et al. Evolution des activités alimentaires des vaches laitières en début de lactaion et liason avec les quantités d'aliments ingérées. Reproduction, Nutrition and Dévelopment., v.27, n.1, p.67-75, 1987.

DADO, R.G.; ALLEN, M.S. Variation in and relationships among feeding, chewing and drinking variables for lactating dairy cows. Journal of Dairy Science, v.77, n.1, p.132, 1994.

DE BOEVER, J.L.; ANDRIES, D.I.; BRABANDER, D.L. et al. Chewing activity of ruminants as a measure of physical structure - a review of factors affecting it. Animal Feed Science and Technology, v.27, p.281-291, 1990.

DE BOEVER, J.L. Roughage evaluation of maize and grass silage based on chewing activity measurements with cows. Gand, Belgium: University of Gand, 1991. 185p. Tese (Doutorado em Zootecnia) - University of Gand, 1991.

DESWYSEN, A.G.; ELLIS, W.C.; POND, K.R. Interrelationships among voluntary intake, eating and ruminating behavior and ruminal motility of heifers fed corn silage. Journal of Animal Science, v.64, n.6, p.835-841, 1987.

DESWYSEN, A.G.; DUTTILEUL, P.; ELLIS, W.C. Quantitative analysis of nycterohemeral eating and ruminating patterns in heifers with different voluntary intakes and effects of monensin. Journal of Animal Science, v.67, n.10, p.2751-2761, 1989.

DESWYSEN, A.G.; DUTILLEUL, P.A.; GODFRIN, J.P. et al. Nycterohemeral eating and ruminating patterns in heifers fed grass or corn silage: analysis by finite Fourier transform. Journal of Animal Science, v.71, n.10, p.2739-2747, 1993.

DULPHY, J.P. Influence du poids vif et du niveau d'ingestion sur le comportement alimentaire et mérycique du mouton. Annales de Zootechnie, v.20, p.477-486, 1971.

DULPHY, J.P.; REMONND, B.; THERIEZ, M. Ingestive behavior and related activities in ruminants. In: Digestive physiology and metabolism in ruminants. Connecticut, A.V.I., Publ. Co., Inc. Wesport, 1980. p.103-122.

DULPHY, J;P, FAVERDIN, P. L'ingestion alimentaire chez les ruminats: modalités et phénomènes associés. Reproduction, Nutrition and Dévelopment, v.27, n.2, p.129-155, 1987.

FISCHER, V. Efeitos do fotoperíodo, da pressão de pastejo e da dieta sobre o comportamento ingestivo de ruminantes. Porto Alegre: Universidade Federal do Rio Grande do Sul, 1996. 243p. Tese (Doutorado em Zootecnia) - Universidade Federal do Rio Grande do Sul, 1996.

FORBES, J.M. The voluntary food intake of farm animals. Londres: Butterworth and Co, 1986. 206p.

GORDON, J.G. The act of rumination. Journal Agricultural Science, v.50, n.1, p.34-42, 1958.
GORDON, J.G., Mc ALLISTER, I.K. The circadian rhythm of rumination. Journal Agricultural Science, v.74, n.2, p.291297, 1970.

GRANT, R.J., ALBRIGHT, J.L. Feeding behavior and management factors during the transition period in dairy cattle. Journal of Animal Science, v.73, n.12, p.27912803, 1995.

HODGSON, J. Grazing management: science into practice. Inglaterra: Longman Handbooks in Agriculture, 1990. 203p.

JASTER, E.H., MURPHY, M.R. Effects of varying particle size of forage on digestion and chewing behavior of dairy heifers. Journal of Dairy Science, v.66, n.6, p.802-810, 1983.

NATIONAL RESEARCH COUNCIL - NRC. Nutrients requeriments of dairy cattle. Washington: D.C.: National Academy Press, 2001. 57p.

PEARCE, G.R. Rumination in sheep. II. The circadian pattern of rumination. Australian Journal of Agriculture Research, v.16, n.4, p.635-648, 1965.

RAY, D.E.; ROUBICEK, C.B. Behavior of feedlot cattle during two seasons. Journal of Animal Science, v.33, n.1, p.72-76, 1971.

SAS INSTITUTE. Statistical analysis systems user's guide: ETS. versão 6, 1.ed., Cary: 1988a.

SAS INSTITUTE. Statistical analysis systems procedure's guide. versão 6.03, Cary: 1988b.

SAS INSTITUTE. Statistical analysis systems user's guide, versão 6, 4.ed., Cary: 1989.

SHAVER, R.D.; NYTES, A.J.; SATTER, L.D. et al. Influence of forage physical form and feed intake level on particle size of masticates, ruminal digesta and feces of dairy cows. Journal of Animal Science, v.61(suppl.1), p.480, 1985.

ULYATT, M.J.; DELLOW, D.W.J.; JOHN, A. et al. Contribution of chewing during eating and rumination to the clearence of digesta from the reticulum-rumen. In: Control of digestion and metabolism in ruminants. New Jersey: Englewod Cliffs, 1986. p.498-515.

WELCH, J.G.; SMITH, A.M. Influence of forage quality on rumination time in sheep. Journal of Animal Science, v.28, p.813-818, 1969.

WELCH, J.G.; SMITH, A.M. Forage quality and rumination time in cattle. Journal of Dairy Science, v.53, p.797-800, 1970.

WELCH, J.G.; SMITH, A.M. Physical stimulation of rumination activity. Journal of Animal Science, v.33, p.1118-1123, 1971. 